\title{
Modelling of scanning pulsed eddy current testing of normal and slanted surface cracks
}

\author{
Faris Nafiah", Ali Sophian², Md Raisuddin Khan ${ }^{3}$, Ilham Mukriz Zainal Abidin ${ }^{4}$ \\ ${ }^{1,2,3}$ International Islamic University Malaysia, Malaysia \\ ${ }^{4}$ Leading Edge NDT, Malaysian Nuclear Agency, Malaysia
}

\begin{abstract}
Article Info
ABSTRACT

Article history:

Received Sep 26, 2018

Revised Feb 22, 2019

Accepted Jun 1, 2019

Thanks to its wide bandwidth, pulsed eddy current (PEC) has attracted researchers of various backgrounds in the attempt to exploit its benefits in Non-destructive Testing (NDT). The ability of modelling PEC problems would be a precious tool in this attempt as it would help improve the understanding of the interaction between the transient magnetic field and the specimen, among others. In this work, a Finite Element Modelling (FEM) has been developed and experimental test data have been gathered for its validation. The investigated cases were simulated surface cracks of different sizes and angles. The study involved looking at time-domain PEC signals at different spatial distances from the cracks' faces, which would particularly be useful for modelling scanning PEC probes. The obtained results show a good agreement between the FEM and experiment, demonstrating that the modelling technique can be used with confidence for solving similar problems. In addition, the extracted features from signals were also studied to discover the influence of crack geometries to the PEC responses.
\end{abstract}

Copyright (C) 2019 Institute of Advanced Engineering and Science. All rights reserved.

\section{Corresponding Author:}

Ali Sophian,

International Islamic University Malaysia,

Jalan Gombak, 53100, Kuala Lumpur, Malaysia.

Email: ali_sophian@iium.edu.my

\section{INTRODUCTION}

Material integrity assessment during manufacturing and in-service is commonly performed to evaluate the integrity and reliability of structures and parts [1,2]. Such assessments can be achieved by using non-destructive testing (NDT) techniques, which enable the operators to detect or evaluate defects in the object without causing any permanent damage to it. Eddy current testing (ECT) is one of the techniques employed in NDT $[3,4]$. Falling under ECT, pulsed eddy current (PEC) utilizes magnetic field to be directed into a conductive tested sample for detection, characterization and quantification of the flaws . While conventional ECT employs only a single excitation frequency, PEC offers the advantage of impinging a broad spectrum of excitation frequencies, which potentially provide more information regarding the flaws [2, 4-6].

Typical PEC signals are reference, response and differential signals [1], where reference signal is the signal obtained from an unflawed sample, response signal is the flaw signal, while subtracting reference signal from the response signal gives the differential signal. In time domain, the peak value and peak arrival time of the differential signal are the most common features used [4].

PEC problem has been approached in different ways, i.e. analytically [7-11], numerically [12, 13], and experimentally [14]. Literature shows that numerical solution, such as finite element modelling [15-17] attracts a lot of interest among researchers, as it monitors the physical phenomenon and provides a clear visual presentation. In a bid to exploit those advantages, FEM was used to find the optimal probe design for coaxially coupled and driving pickup coil [18]. FEM has also been applied to find the ideal rectangular coil dimensions for conductivity evaluation [19]. Aside from that, generated estimates from FEM have also been used to be 
compared with developed analytical solutions to model the transient response of an encircling coil around a ferromagnetic conducting rod [20]. Researchers also made the most of the axisymmetric design, employing only a quarter of the geometry to investigate the effects of electrical run-out and the electromagnetic property of the sample [21].

Complex PEC probe designs can also be modelled as FEM software supports CAD kernel designs [16]. Additionally, FEM also offers flexibility in studying pick-up sensors allowing the use of sensor array [22], for example. Moreover, it also allows researchers to investigate various effects of coil design to depth of penetration of PEC, while readily monitoring the eddy current density in the sample [23].

Looking at various potential applications of FEM in PEC, it is undoubtedly necessary to study the correlation between FEM simulation and experimental results. Previous recorded research only compares or validates the trends of the obtained results from simulation and experiments, using either normalised signals or simply observing at the behaviour of magnetic flux density measured at different conditions, while ignoring the absolute value of the magnetic flux density measured. On top of that, some others also do not include different positioning of the coil probe. In this paper, the authors propose to compare solving PEC problems using FEM against experimental analysis for a set of simulated cracks and coil positions viz-a-viz: signals' nominal values. This paper also observes the spatial trends of peak value and peak arrival time extracted from each signal at different positions to reveal the influence of crack sizes and angles. Precautionary steps to achieve optimal comparisons between FEM and experimental results are presented, accompanied with the measure of comparisons for both approaches.

\section{RESEARCH METHOD}

Three surface cracks were analysed that can be divided into two groups, namely normal and slanted cracks. Normal cracks of different depths were studied, $6 \mathrm{~mm}$ and $8 \mathrm{~mm}$. The slanted crack is of $60^{\circ}$ angle to the sample surface, and $8 \mathrm{~mm}$ vertical depth. Crack widths, illustrated in Figure 1, are created constant at 0.32 $\mathrm{mm}$ by using an electric discharge machine (EDM) into $10 \mathrm{~mm}$-thick aluminium slabs. The middle of the crack face is assigned as $0 \mathrm{~mm}$ position, while the left-hand and right-hand sides of the crack are assigned as negative and positive positions respectively. The analysed positions for normal crack were $0 \mathrm{~mm}, 1 \mathrm{~mm}$, $3 \mathrm{~mm}, 5 \mathrm{~mm}$ and $8 \mathrm{~mm}$, while for slanted crack, the coil is placed at $-2 \mathrm{~mm},-1 \mathrm{~mm}, 0 \mathrm{~mm}, 1 \mathrm{~mm}$, and $2 \mathrm{~mm}$.
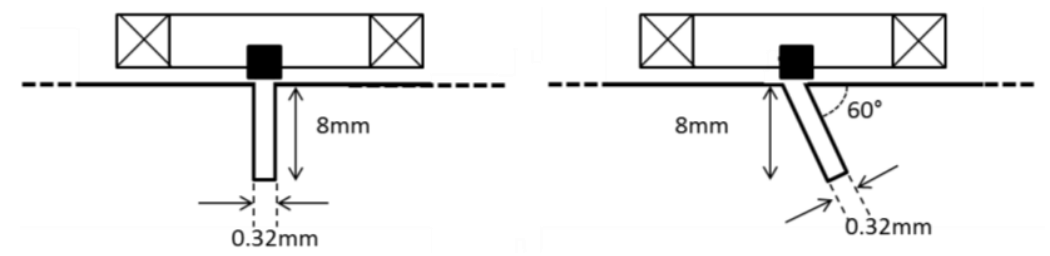

Figure 1. Manufactured cracks (a) $8 \mathrm{~mm}$ depth normal crack while (b) 60deg $8 \mathrm{~mm}$ vertical depth stanted crack

\subsection{Finite Element Modelling}

A 3D FEM model has been built using COMSOL Multiphysics 5.2a and its AC/DC module to simulate our PEC system. To maximise the agreement between FEM and experimental results, careful consideration on the parameters and loading conditions was done to meet the specifications based on the experimental setup. The aluminium samples were analysed under a measuring microscope with a $20 \mathrm{X}$ eyepiece to find a width of $0.331 \mathrm{~mm}$ and depth of $5.947 \mathrm{~mm}$ for $6 \mathrm{~mm}$ normal crack and $0.318 \mathrm{~mm}$ width and $7.804 \mathrm{~mm}$ depth for $8 \mathrm{~mm}$ normal crack. The slanted crack has a vertical depth of $7.985 \mathrm{~mm}$ and an angle of $59.54^{\circ}$. The electrical conductivity was set at $25.1 \times 106 \mathrm{~S} / \mathrm{m}$ for the sample whose dimensions were set to be $150 \times 75 \times 10 \mathrm{~mm}$, which is more than three times larger than the largest geometric dimensions of the coil, to avoid the edge effect [24]. The air domain dimension was also set as a cuboid of dimension of more than three times the diameter of the coil to approach an infinite domain [25]. A coil with inner and outer radii of 6.5 and $10.05 \mathrm{~mm}$ respectively was positioned at a 1-mm lift-off and the point to evaluate the picked-up magnetic flux density was at $0.5 \mathrm{~mm}$ above the surface of the sample as obtained from the SS4951A datasheet. Curvefitting was done in Matlab to obtain the 2nd order expression of the excitation current in the experiment, consequently transferred to the FEM. The boundaries of the air domain were modelled to be magnetically insulated to ensure no flux diverged outside of the domain of interest. The FEM was computed with time-

Indonesian J Elec Eng \& Comp Sci, Vol. 16, No. 3, December 2019 : 1297 - 1302 
dependent study mode, starting at 0 up to $5 \mathrm{~ms}$, with nine logarithmically spaced-out values in-between. The results were later spline-interpolated.

\subsection{Mesh Convergence Analysis}

Mesh convergence analysis was done with five different mesh configurations as shown in Table 1, using 3D model FEM where the coil is positioned at the middle of sample without flaws (reference signal). The model was generated with coarsest mesh and repeated while iterating the mesh refinement. Transient signals of the magnetic flux density were later analysed and compared to the finest in 2D axisymmetric model, as it offers much finer mesh with much lower computation time. The percentage of similarity to $2 \mathrm{D}$ axisymmetric was calculated as

$$
\% \text { of Similarity }=100 \%-\frac{\sum_{n=0}^{N} \frac{\left|X_{3 D}(n)-X_{2 D}(n)\right| \times 100 \%}{X_{2 D}(n)}}{N}
$$

where $\mathrm{X} 3 \mathrm{D}$ is the transient response obtained from 3D model of different mesh, $\mathrm{X} 2 \mathrm{D}$ is the transient response obtained from 2D axisymmetric model, $\mathrm{N}$ is the total number of points.

It was found that the model stops converging at 'Finer' mesh configuration with a deviation of $0.44 \%$ from the axisymmetric model. 'Extra fine' mesh configuration was chosen as it shows a good compromise between accuracy as well as the computation time, as shown in Table 1.

Table 1. Mesh Configurations

\begin{tabular}{|c|c|c|c|c|c|c|c|}
\hline Mesh type & Extrem. Fine & Extra Fine & Finer & Fine & Normal & Coarse & Coarser \\
\hline Total mesh element & 1057327 & 495627 & 162556 & 43415 & 19696 & 9713 & 5549 \\
\hline Computation times, $\mathrm{s}$ & 12855 & 5647 & 1582 & 468 & 141 & 100 & 66 \\
\hline Similarity with finest mesh, $\%$ & 99.58 & 99.69 & 99.56 & 98.39 & 97.44 & 91.18 & 86.62 \\
\hline
\end{tabular}

Consequently, from the obtained mesh information, the FEM results were computed. For every position of the coil, both response and reference signal were obtained to eliminate error due to meshing. To do this, the material of the crack was either assigned as air for reference signal or aluminium for response signal.

\subsection{Experimental Setup}

The built PEC system comprises of an excitation current circuit, an excitation coil, a sensor, a data acquisition system (DAQ), and a 3D-scanner. A 9-A excitation current was used to induce a strong magnetic field into the sample to achieve high sensitivity, without getting excessive of the sensor's sensing range. Parameters of the excitation coil were attentively taken into consideration in order to get the desired output signal. A $0.35-\mathrm{mm}$-diameter wire was wound around a coil former, forming a coil with inner and outer radii of 6.5 and $10.04 \mathrm{~mm}$ respectively. The outer diameter was made to be smaller than the size of the sample by a factor of three to avoid edge effect, while the inner diameter was chosen to be small to optimize the amount of energy delivered to the sample. The number of turns is 81 , which is low enough to avoid high inductance in the coil, which will undesirably reduce the rising rate of the excitation. The height of the coil was selected to be as small as possible, $3.13 \mathrm{~mm}$, to ensure optimum magnetic coupling while maintaining equal adjacent sides of the coil wound. The lift-off was $1 \mathrm{~mm}$. The 3D scanner was used to change the position the probe relative to the crack.

A SS495A1 Hall device was placed in the middle of probe for sensing the magnetic field. Its output signal is acquired by using NI USB-6363 DAQ system with a set sampling rate of $100 \mathrm{kS} / \mathrm{s}$. The rising edge of the excitation pulse control signal was used as the trigger with 30 pre-trigerred data points.

One-line scanning perpendicular to the crack was carried out at $0.5 \mathrm{~mm}$ resolution. For each spatial position, five pulses were supplied and five-pulse signals were obtained. The signals were then averaged to reduce the noise before being converted to magnetic flux density using the sensitivity of the Hall device. The 30 samples of pre-triggered signals were averaged and used as an offset reference value. The signals acquired were also spline-interpolated to $0.1 \mathrm{~mm}$ resolution.

In the absence of position encoder, the signals acquired were positioned in such a way that the signal with maximum peak value extracted was assigned as the $0 \mathrm{~mm}$ position. For the slanted crack, the position with the maximum peak value, was assigned as the $0.5 \mathrm{~mm}$ position instead, as observed in FEM and explained in Section 3.2 


\section{COMPARISON RESULTS AND DISCUSSIONS}

\subsection{Validation of FEM Results with Experiments}

From the plot of differential signal in Figure 2, it is shown that FEM shows a good agreement with the experiment, where the curves can be identified as almost similar. Some FEM curves are not very visible due to the high similarities. As expected, the further the coil from the middle of the defect, the lower the differential signal. This corresponds to slower rising time of the response signal, because the eddy current is less perturbed by the presence of air.

The two features extracted from both FEM and experiment can be seen in Figure 3 and Figure 4, which show a good agreement between the experiment and FEM. The discrepancy errors in the peak arrival times, which range from $0-18.2 \%$, are relatively higher than those in the peak values, which range from $0.7-$ $15.9 \%$. This is thought to be caused by the relatively large time step, which is $0.01 \mathrm{~ms}$.

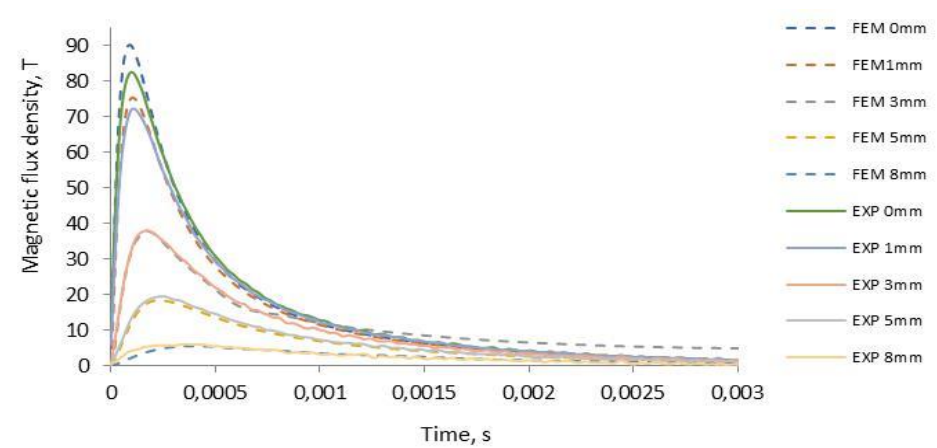

Figure 2. Differential signals for $8 \mathrm{~mm}$ crack, at different spatial positions

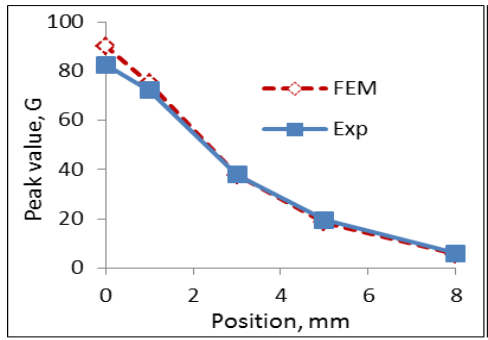

(a)

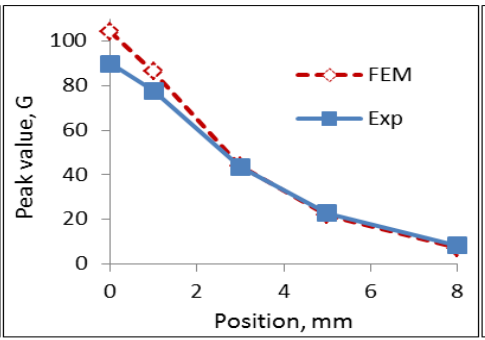

(b)

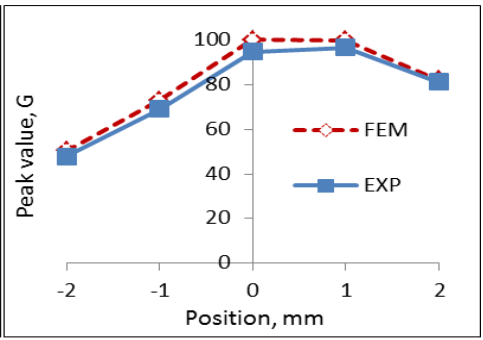

(c)

Figure 3. Peak values vs. positions for (a) $6 \mathrm{~mm}$-deep normal crack, (b) $8 \mathrm{~mm}$-deep normal crack, and (c) slanted crack

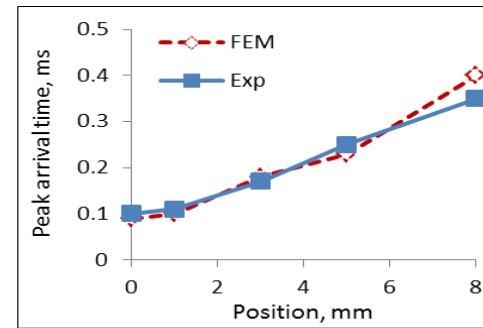

(a)

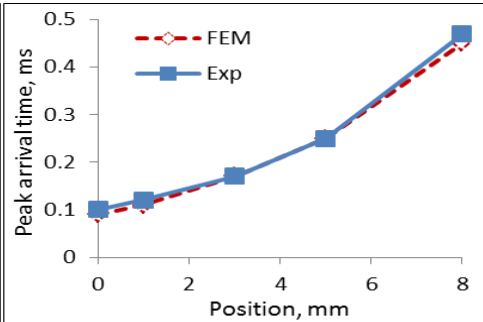

(b)

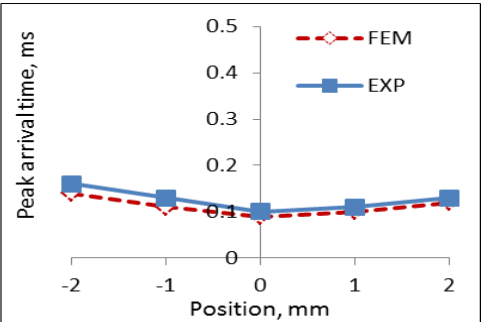

(c)

Figure 4. Peak arrival times vs. positions for (a) 6mm-deep normal crack, (b) 8mm-deep normal crack, and (c) slanted crack

The trends of the features relative to their position show that the different natures of the cracks result in different trends. For normal cracks, we can see a symmetrical trend of the features, relative to the probe distance from the middle of the crack. Meanwhile, the slanted crack displays a skewed trend, where the skewness is relative to the effect of cracks on the probe positions. 


\subsection{Positioning of Maximum Peak Values of Slanted Crack}

Using FEM, the differential signals were obtained at position $-2 \mathrm{~mm}$ up until $5 \mathrm{~mm}$ across the sample at a resolution of $1 \mathrm{~mm}$, and the signals were spline-interpolated to a resolution of $0.1 \mathrm{~mm}$. The maximum peak value found for slanted crack was found to be at position $0.5 \mathrm{~mm}$ instead of $0 \mathrm{~mm}$. This is thought to be due to the volume of crack present at the respective positions.

To study the geometry of the volume of crack, the cross-sectional area of the cracks as seen at different positions, on 2D geometry are analysed. For each position, $0.25 \mathrm{~mm}$ spans on both left and right sides are assigned as the region of interest of the area of the crack. Figure 5 shows that position $0 \mathrm{~mm}$ has a crosssectional crack area of a $0.16 \mathrm{~mm} 2$ trapezium, while position $0.5 \mathrm{~mm}$ has a bigger cross-sectional crack area of $0.32 \mathrm{~mm} 2$ parallelogram.

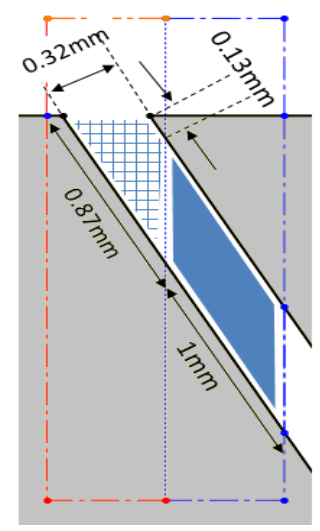

Figure 5. Cross-sectional area of the slanted crack at $0 \mathrm{~mm}$ (gridded), and position $0.5 \mathrm{~mm}$ (solid colour)

\subsection{C-scan Images of Different Cracks}

C-scan images of dimension of $4 \times 4 \mathrm{~mm}$ were obtained for each crack by interpolating and duplicating the peak values from position -2 to $2 \mathrm{~mm}$. The small scanning area was chosen to avoid false interpretation due to the possible neighbouring crack present in real-life applications. The $\mathrm{C}$-scan image for the slanted crack shows a negatively skewed image, while both normal cracks give a symmetrical image, as seen in Figure 6. Post-processing can be done on the images to obtain features to differentiate between normal and slanted crack, while different angles can be further simulated in future works to quantify the crack angles, based on their C-scan images

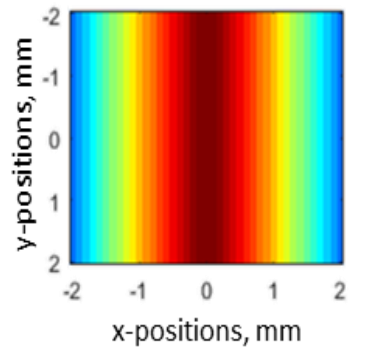

(a)

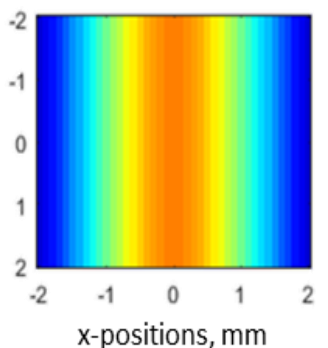

(b)

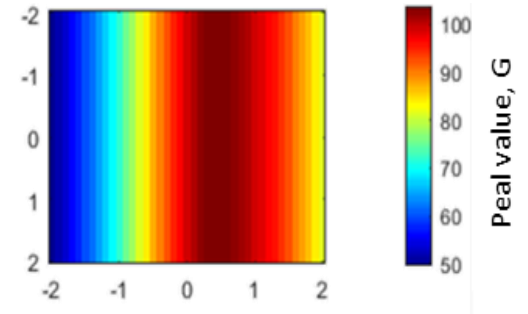

(c)

Figure 6. C-scan images of (a) normal crack $8 \mathrm{~mm}$ depth, (b) normal crack $6 \mathrm{~mm}$ depth and (c) slanted crack

\section{CONCLUSIONS}

The paper presents the steps in building a finite element model of PEC that has a good correlation with experimental results; not just in the trends, but also in the nominal values obtained. It shows that FEM has been successfully employed to obtain strong correlation with most of the discrepancy errors are within the range of $10 \%$. As mentioned above, owing to the lack of encoder to the built PEC system, FEM also offers positioning of the differential signal with respect to the coil position. These advantages are in favour of the current research being undertaken, utilizing FEM to extract features to differentiate natures of cracks,

Modelling of scanning pulsed eddy current testing of normal and slanted surface cracks (Faris Nafiah) 
as well as studying the shift of the maximum peak value in C-scan images. The paper also presents the different spatial trends of features of slanted cracks as compared to normal cracks. The features are dependent on the probe distance to the most crack-affected area. The normal crack displays a symmetrical trend, while a skewed trend is seen on the slanted crack. In future work, the model and findings will be used in the research on imaging of surface and sub-surface defects of different characteristics cracks.

\section{ACKNOWLEDGEMENTS} 059-0558.

The work was supported by the Malaysia's Ministry of Higher Education through the grant FRGS16-

\section{REFERENCES}

[1] G. Y. Tian and A. Sophian, "Defect classification using a new feature for pulsed eddy current sensors," $N D T \& E$ International, vol. 38, no. 1. pp. 77-82, 2005.

[2] Y. He et al., "Pulsed eddy current technique for defect detection in aircraft riveted structures," NDT E Int., vol. 43, no. 2, pp. 176-181, Mar. 2010.

[3] S. K. Dwivedi, M. Vishwakarma, and P. A. Soni, "Advances and Researches on Non Destructive Testing: A Review," Mater. Today Proc., vol. 5, no. 2, Part 1, pp. 3690-3698, 2018.

[4] A. Sophian, G. Y. Tian, D. Taylor, and J. Rudlin, "Electromagnetic and eddy current NDT: A review," Insight NonDestructive Test. Cond. Monit., vol. 43, no. 5, 2001.

[5] N. Ulapane, A. Alempijevic, T. Vidal Calleja, and J. Valls Miro, "Pulsed Eddy Current Sensing for Critical Pipe Condition Assessment," Sensors, vol. 17, no. 10, p. 2208, 2017.

[6] Z. Xu, X. Wu, J. Li, and Y. Kang, "Assessment of wall thinning in insulated ferromagnetic pipes using the time-topeak of differential pulsed eddy-current testing signals," NDT E Int., vol. 51, pp. 24-29, Oct. 2012.

[7] M. Fan, P. Huang, B. Ye, D. Hou, G. Zhang, and Z. Zhou, "Analytical modeling for transient probe response in pulsed eddy current testing," NDT E Int., vol. 42, no. 5, pp. 376-383, Jul. 2009.

[8] X. Chen and Y. Lei, "Time-domain analytical solutions to pulsed eddy current field excited by a probe coil outside a conducting ferromagnetic pipe," NDT E Int., vol. 68, pp. 22-27, Dec. 2014.

[9] M. . Fan, B. . Cao, and Y. . Wang, "Computation of coil-induced voltage due to a defect-free plate using Stehfest's method for pulsed eddy current evaluation," Insight Non-Destructive Test. Cond. Monit., vol. 52, no. 6, pp. 302-304, 2010.

[10] X. Chen and Y. Lei, "Electrical conductivity measurement of ferromagnetic metallic materials using pulsed eddy current method," NDT E Int., vol. 75, pp. 33-38, Oct. 2015.

[11] T. Theodoulidis, H. Wang, and G. Y. Tian, "Extension of a model for eddy current inspection of cracks to pulsed excitations," NDT E Int., vol. 47, pp. 144-149, Apr. 2012.

[12] S. x. Liu, W. Xin, and K. q. Ding, "Simulation of corrosion on detection for pulsed eddy current," Fuzzy Systems and Knowledge Discovery (FSKD), 2010 Seventh International Conference on, vol. 4. pp. 1839-1842, 2010.

[13] J. Pavo, "Numerical calculation method for pulsed eddy-current testing," IEEE Transactions on Magnetics, vol. 38, no. 2. pp. 1169-1172, 2002.

[14] A. Sophian, G. Tian, and M. Fan, "Pulsed Eddy Current Non-destructive Testing and Evaluation: A Review," Chinese J. Mech. Eng., vol. 30, no. 3, pp. 500-514, 2017.

[15] V. K. Babbar, P. R. Underhill, C. Stott, and T. W. Krause, "NDT \& E International Finite element modeling of second layer crack detection in aircraft bolt holes with ferrous fasteners present," NDT E Int., vol. 65, pp. 64-71, 2014.

[16] V. K. Babbar, P. V. Kooten, T. J. Cadeau, and T. W. Krause, "Finite element modeling of pulsed eddy current signals from conducting cylinders and plates," AIP Conf. Proc., vol. 1096, pp. 311-318, 2009.

[17] B. Allen, N. Ida, and W. Lord, "Finite element modeling of pulse Eddy current NDT phenomena," IEEE Transactions on Magnetics, vol. 21, no. 6. pp. 2250-2253, 1985.

[18] J. A. Buck et al., "Pulsed eddy current inspection of support structures in steam generators," IEEE Sens. J., vol. 15, no. 8, pp. 4305-4312, 2015.

[19] D. Zhou, Y. Li, X. Yan, L. You, Q. Zhang, and X. Liu, "The investigation on the optimal design of rectangular PECT probes for evaluation of defects in conductive structures," vol. 42, pp. 319-326, 2013.

[20] D. P. R. Desjardins, T. W. Krause, and N. Gauthier, "Analytical modeling of the transient response of a coil encircling a ferromagnetic conducting rod in pulsed eddy current testing," NDT E Int., vol. 60, pp. 127-131, Dec. 2013.

[21] I. D. Adewale and G. Y. Tian, "Decoupling the Influence of Permeability and Conductivity in Pulsed Eddy Current Measurements," IEEE Trans. Magn., vol. 49, no. 3, pp. 1119-1127, 2013.

[22] R. F. Abrantes, L. S. Rosado, M. Piedade, and P. M. Ramos, "Pulsed eddy currents testing using a planar matrix probe,” Meas. J. Int. Meas. Confed., vol. 77, pp. 351-361, 2016.

[23] S. Majidnia, R. Nilavalan, and J. Rudlin, "A numerical study of the depth of penetration of eddy currents," Insight Non-Destructive Test. Cond. Monit., vol. 58, no. 3, pp. 129-134, 2016.

[24] J. García-Martín, J. Gómez-Gil, and E. Vázquez-Sánchez, "Non-destructive techniques based on eddy current testing," Sensors, vol. 11, no. 3, pp. 2525-2565, 2011.

[25] R. S. Wadhwa, "Development of a robust handling model for foundry automation," in Proceedings of the 37th International MATADOR Conference, 2012, vol. 6, p. 215.

Indonesian J Elec Eng \& Comp Sci, Vol. 16, No. 3, December 2019 : 1297 - 1302 